\title{
Numerical method for reducing spurious pressure oscillations in transcritical flow simulations
}

\author{
Bradley Boyd ${ }^{* 1}$, Dorrin Jarrahbashi ${ }^{1}$ \\ 'J. Mike Walker '66 Mechanical Engineering Department, \\ Texas A\&M University, College Station, USA \\ *Corresponding author email: bradley.w.boyd@gmail.com
}

\begin{abstract}
The spurious pressure oscillations are the most common numerical instabilities observed in multiphase and multicomponent flow problems near the critical point. A diffuse-interface model is developed to simulate transcritical mixing in multispecies and multiphase systems where spurious pressure oscillations are problematic. To reduce the spurious pressure oscillations, we developed a hybrid method (HY) that switches between the quasi-conservative double-flux (DF) method and the classical fully-conservative (FC) numerical procedure based on the changes in the effective specific heat ratio and the effective reference internal energy. We consider the transcritical Spray A benchmark case of the Engine Combustion Network (ECN) that represents the injection of $n$-dodecane into a high-pressure and high-temperature nitrogen environment. The HY model is found to effectively reduce the magnitude of the spurious pressure oscillations compared to the FC method, and reduce the loss of energy conservation compared to the DF method.
\end{abstract}

\section{Keywords}

Spurious pressure oscillations; Diffuse-interface methods; Transcritical Flow; Peng-Robinson Equation of State.

\section{Introduction}

Simulating transcritical multi-flow problems is critical for fuel injection applications including diesel and rocket engines [1-5]. In the case of a diesel engine, the diesel is injected into a high-pressure-temperature environment of air where the pressure is above the critical pressure of the fuel. As the diesel jet mixes with the air, the fuel temperature increases past its critical temperature, thus transcritical mixing occurs. Many studies have reported the challenges in alleviating the spurious pressure oscillations to achieve numerical stability at transcritical conditions [6-10]. A summary of the major numerical solvers used in the literature for simulating transcritical flow problems has been summarized by Oefelein et al. [9].

A common way to reduce the magnitude of spurious pressure oscillations is to utilize numerical diffusion either by artificial viscosity [11, 12] or low-order reconstruction and flux approximations like the entropy-stable hybrid scheme described in [8]. Alternatively, quasiconservative methods (QC) were developed to mitigate pressure oscillations either using the pressure evolution equation $[12,13]$ or the double-flux (DF) method [8]. The problem with these QC methods is the loss of energy conservation results in the heat-up of the fuel in the n-dodecane spray case [7] and the incorrect prediction of the shock speed and temperature distribution in shock-tube problems [13]. Another QC method recently considered is an enthalpy-based approach, which does a better job of conserving energy; however, in a shocktube case, it still suffers from spurious pressure oscillations and incorrectly predicts the shock speed [13].

We aim to develop a numerical method to reduce the spurious pressure oscillations while maintaining a high-order reconstruction and reducing or eliminating the loss of energy 
conservation. In this study, we present a numerical method that is a hybrid between the fullyconservative method (FC) and the quasi-conservative double-flux method (DF). This hybrid method $(\mathrm{HY})$ uses the DF for finite volume cells where pressure oscillations are expected to occur while using the FC method elsewhere to reduce the overall loss of energy conservation. The HY method is compared to the FC and DF throughout this work. The effectiveness of the HY method is showcased using the transcritical mixing of an n-dodecane jet into a nitrogen environment.

\section{Material and Methods}

We consider a compressible, inviscid, multiphase model, with multiple fluid species. The governing equations for a two-species system are

$$
\begin{gathered}
\frac{\partial(\rho)}{\partial t}+\nabla \cdot(\rho \mathbf{u})=0 \\
\frac{\partial\left(\rho Y_{1}\right)}{\partial t}+\nabla \cdot\left(\rho \mathbf{u} Y_{1}\right)=0 \\
\frac{\partial(\rho \mathbf{u})}{\partial t}+\nabla \cdot(\rho \mathbf{u} \otimes \mathbf{u}+p \mathbf{I})=\mathbf{0} \\
\frac{\partial E}{\partial t}+\nabla \cdot(\mathbf{u}(E+p))=0,
\end{gathered}
$$

where $Y_{1}$ is the mass fraction of the first fluid species (nitrogen), $\rho$ is the density, $\mathbf{u}$ is the velocity vector, $p$ is the pressure, $E$ is the total energy $E=\rho\left(e+|\mathbf{u}|^{2} / 2\right), e$ is the internal energy, and $\mathbf{I}$ is the identity matrix. The mass fraction of the second species (n-dodecane) of the two-component system is given by the mixture rule $Y_{2}=1-Y_{1}$. The system of equations, Eqs. (1)-(4), is closed using the Peng-Robinson equation of state (PR-EoS)

$$
p=\frac{R T}{V_{m}-b}-\frac{a}{V_{m}^{2}+2 b V_{m}-b^{2}},
$$

where $R$ is the universal gas constant, $V_{m}$ is the molar volume $V_{m}=M / \rho, M$ is the molar mass, and $a$ and $b$ are coefficients that depend on the state and composition of the fluid. The mixture rules for determining the thermodynamic quantities are presented in the work by $\mathrm{Ma}$ [1].

For this study, we neglect surface tension, viscosity, heat conduction, and the interfacial diffusion term. These phenomena may be of importance for transcritical problems; however, they are not the cause of spurious pressure oscillations. Thus, they provide no additional insight into the focus of this study which is reducing the spurious pressure oscillations. The diffuse terms of heat, species mass fraction, and viscosity act to reduce the magnitude of pressure fluctuations. It is important to note that the addition of these terms to the proposed numerical framework presented here is very straightforward and has been done previously [1, 2]. The influence of surface tension is typically neglected for transcritical flow models [1-11].

In the current study, we consider the 2D injection of $n$-dodecane into nitrogen. The numerical scheme uses the fifth-order weighted essentially non-oscillatory (WENO5) reconstruction and the Harten-Lax-van Leer-contact (HLLC) approximate Riemann flux [12-14]. The temporal discretization of Eqs. (1)-(4) uses the third-order total-variation-diminishing Runge-Kutta 
scheme. Additionally, we make use of a maximum-principle satisfying and positivity preserving flux limiter to help ensure the boundness of the mass fraction and the positivity of density and pressure [13-15].

Typically, the reconstruction procedure is done on the primitive variables. Another approach to reduce spurious pressure oscillations is to reconstruct the primitive variables in characteristic space, referred to as the characteristic variables. This approach avoids the interaction of the different characteristic fields [16]. To reconstruct the characteristic variables, a local characteristic decomposition is first performed [16-19].

We also modify the PR-EoS because under certain conditions in the vapor dome the speed of sound may become complex-valued or the pressure may become negative - see [2] for more details. The PR-EoS is modified following the procedure presented in [20], where the pressure is given by an approximate saturation pressure of the mixture if the state is within the vapor dome. The modified PR-EoS [20] also limits the speed of sound to a minimum value - the minimum value speed of sound used in this paper is $1 \mathrm{~m} / \mathrm{s}$. This method is the simplest modification to the PR-EoS to ensure the robustness of the solver if the state falls inside the vapor dome. If more accurate results are required when significant phase-separation is anticipated [11], the VLE method presented in [2] is a more accurate alternative for determining the saturation pressure and the speed of sound of the mixture. For this study, however, the numerical procedure of [20] is sufficient for studying the pressure oscillations of transcritical flow.

The DF method differs from the classical FC procedure for solving the Euler equations. The key difference is that the DF method uses the effective specific heat ratio $\left(\gamma^{*}\right)$ and the effective reference internal energy $\left(e_{0}^{*}\right)$ from the previous time step to relate internal energy to pressure [21]

$$
p=\frac{1}{\Gamma^{*}}\left(\rho E-\rho e_{0}^{*}-\frac{1}{2} \rho|\mathbf{u}|^{2}\right)
$$

where

$$
\Gamma^{*}=\frac{1}{\gamma^{*}-1}, \gamma^{*}=\frac{\rho c^{2}}{p}, e_{0}^{*}=e-\Gamma^{*} \frac{p}{\rho}
$$

Whereas, the FC method uses the PR-EoS to directly relate internal energy to pressure. We refer the reader to Figure 1 of Ref [22] for more details on the time advancement steps of the DF and FC methods.

It was presented in $[1,23]$ that the change in $\Gamma^{*}$ and $e_{0}^{*}$ between cells results in the loss of pressure equilibrium in the numerical scheme and is, therefore, the primary source of spurious pressure oscillations. To more robustly capture a range of problems, we developed a hybrid combination of the FC method and the DF method. The hybrid method (HY) determines the jump in $\Gamma^{*}\left(\Delta \Gamma^{*}\right)$ and $e_{0}^{*}\left(\Delta e_{0}^{*}\right)$ between the cell and its neighbours, for example, the maximum $\Delta \Gamma^{*}$ and $\Delta e_{0}^{*}$ for a cell in one-dimensional Cartesian coordinates are defined as

$$
\begin{aligned}
& \Delta \Gamma_{i, \max }^{*}=\max \left(\left|\Gamma_{i+1}^{*}-\Gamma_{i}^{*}\right|,\left|\Gamma_{i}^{*}-\Gamma_{i-1}^{*}\right|\right), \\
& \Delta e_{0, i, \max }^{*}=\max \left(\left|e_{0, i+1}^{*}-e_{0, i}^{*}\right|,\left|e_{0, i}^{*}-e_{0, i-1}^{*}\right|\right),
\end{aligned}
$$


where $i$ refers to the cell index. If $\Delta \Gamma_{i, \max }^{*}$ or $\Delta e_{0, i, \max }^{*}$ is above the corresponding tolerances $\Delta \Gamma_{i, t o l}^{*}, \Delta e_{0, i, t o l}^{*}$ then the DF method is used for determining the flux in the cell, otherwise, the FC flux is used. We determined, by numerical experiments using different fuels (n-dodecane, $\mathrm{n}$-heptane, and ethane), that $\Delta \Gamma_{i, \text { tol }}^{*}=1$ and $\Delta e_{0, i, \text { tol }}^{*}=1000[\mathrm{~kJ} / \mathrm{kg}]$ yield an acceptable balance between stability and accuracy.

A similar hybrid method was recently developed in [24], where they switched between the DF and FC method depending on the density gradients, i.e. $|\nabla \rho| / \rho$. They used the DF method if $|\nabla \rho| / \rho \geq 0.3$ and the FC method when $|\nabla \rho| / \rho<0.3$. This method works equally well at identifying the interface between different species and phases where the pressure oscillations originate in transcritical problems. However, if we consider a case where the effective internal energy or effective ratio of specific heats changes significantly between two species, i.e. a large $\Delta \Gamma_{i \text {,max }}^{*}$ or $\Delta e_{0, i, \max }^{*}$, but the density does not vary significantly, the $\mathrm{HY}$ method with $\Delta \Gamma_{i, \text { max }}^{*}$ and $\Delta e_{0, i, \max }^{*}$ switch would be more effective at reducing the spurious pressure oscillation than the $|\nabla \rho| / \rho$ switch. Additionally, Ma et al. [25] briefly considered using a switch condition based on $\gamma_{\text {tol. }}^{*}=8$; however, this method does not consider changes in $e_{0}^{*}$.

Additionally, we initially use interface thickening of the diffuse-interface between the fluids. For example, in the n-dodecane jet simulation, the interface of the jet is smeared over two finite volume cells (Figure 1). This reduces the jump in properties across the interface, increasing stability and reducing energy conservation loss [22].

\section{Results and Discussion}

We consider the injection of $n$-dodecane into a nitrogen environment, representing similar conditions to the ENC Spray A case $[3,21]$. This 2D multi-species transcritical flow problem is known to exhibit spurious pressure oscillations [3,21], therefore it is an appropriate test case to evaluate the stability and robustness of the numerical model presented here. The $\mathrm{n}$ dodecane is injected at $363 \mathrm{~K}$ and a velocity of $100 \mathrm{~m} / \mathrm{s}$ into the nitrogen environment, which is at $900 \mathrm{~K}$ and $6 \mathrm{MPa}$ [21]. The bottom boundary is a reflective boundary, the left and right boundaries are periodic, and the top outlet is a transmissive boundary as shown in Figure 1 . 


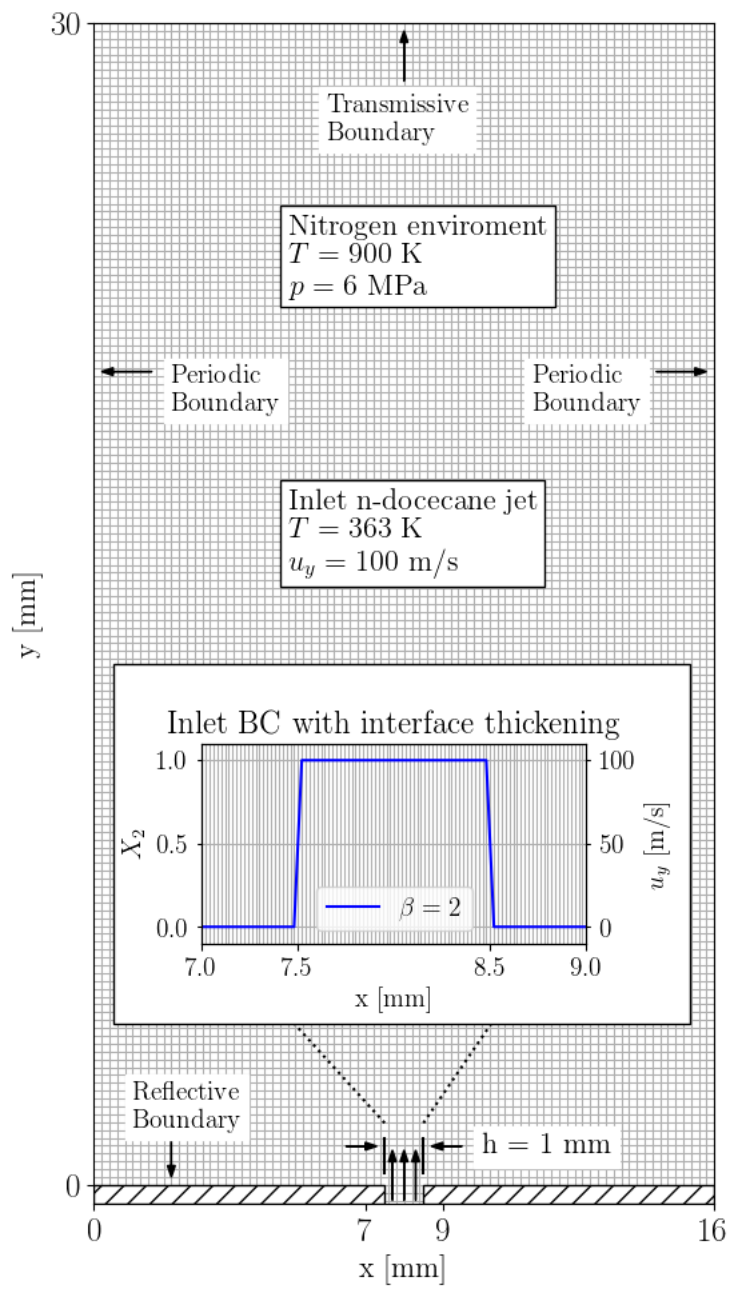

Figure 1. Schematic of the injection of $n$-dodecane into a nitrogen environment described in [21] to represent the ECN Spray A case. The cell size depicted is five times larger than the cell size used in the simulation $(\Delta z \approx \Delta r \approx 0.02[\mathrm{~mm}])$. Note that $X_{2}$ is the molar fraction of $\mathrm{n}$-dodecane and $\beta$ is the number of cells the interface is thickened over.

The transcritical breakup and mixing of the jet, shown by the density and mass fraction distributions in Figure 2, is very similar to the previous results of Ma [21]. The most significant difference between the numerical methods is the temperature distribution (Figure 2(g)-(i)). The DF method results in a significantly higher temperature distribution after $360 \mu$ s (Figure 2(g)) when compared to the FC method (Figure 2(i)). This is because the DF method does not conserve energy resulting in numerical heating as the fluids mix isochorically [21]. The HY also suffers from loss of energy conservation, as it invokes the double-flux method when pressure oscillations are expected to become trouble-some. As a result, the temperature distribution of $\mathrm{HY}$ is lower than the DF but higher than the FC method.

To visualize the loss of energy conservation due to the DF and HY methods, we compare the total energy of the domain to the FC method in Figure 3. The FC conserves energy; however, the total energy within the domain increases steadily as the n-dodecane is injected. After 360 $\mu \mathrm{s}$, the total energy increases to about $4.5 \mathrm{~kJ}$ for the $\mathrm{FC}$ method. For the quasi-conservative methods (DF and HY), the total energy within the domain increases dramatically to about 8.7 $\mathrm{kJ}$ and $9.4 \mathrm{~kJ}$ for the HY and DF methods, respectively. This dramatic increase in energy due to conservation loss is consistent with the increased temperatures of the HY and DF methods presented in Figure 2. 


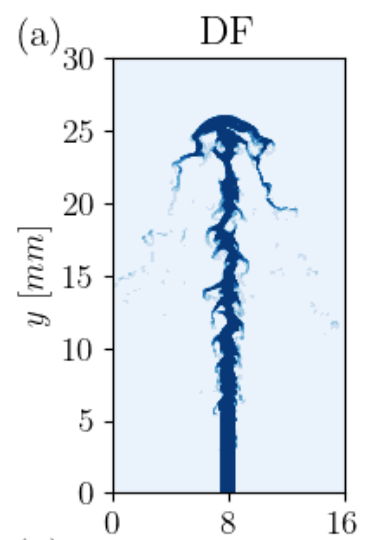

(d)

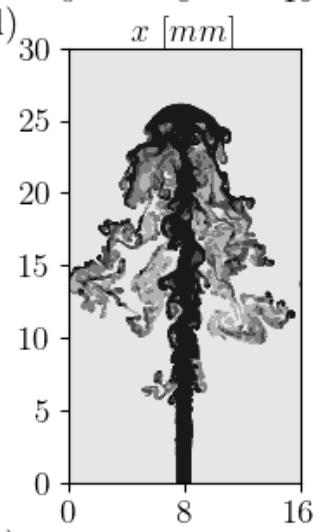

(g)

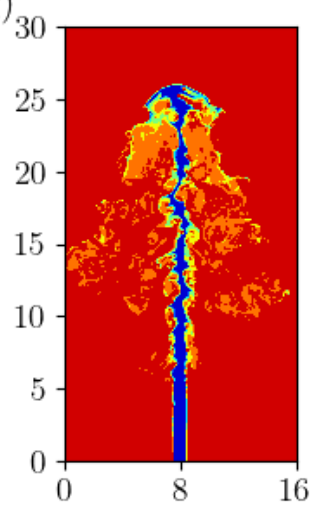

(b)

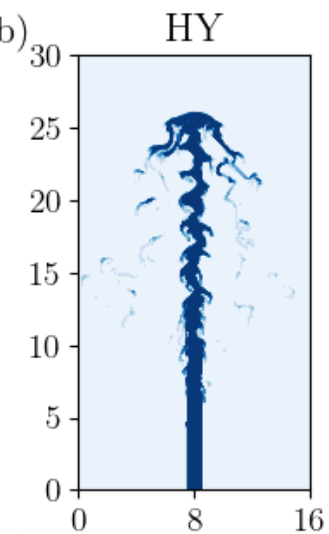

(e)

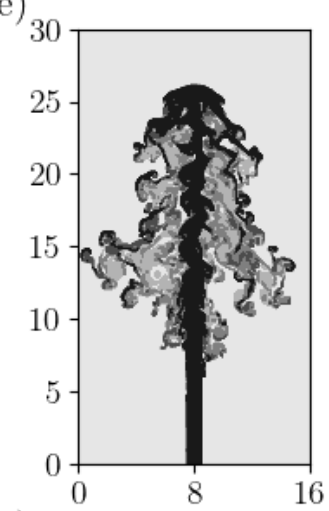

(h)

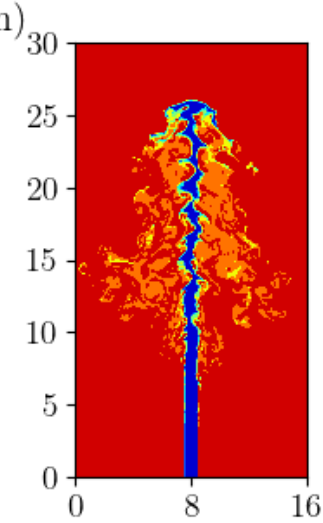

(c)

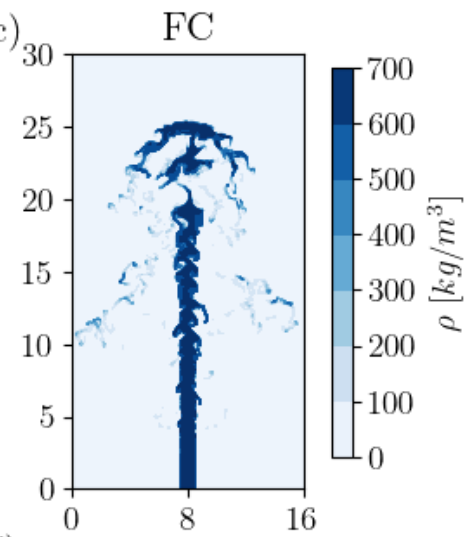

(f)

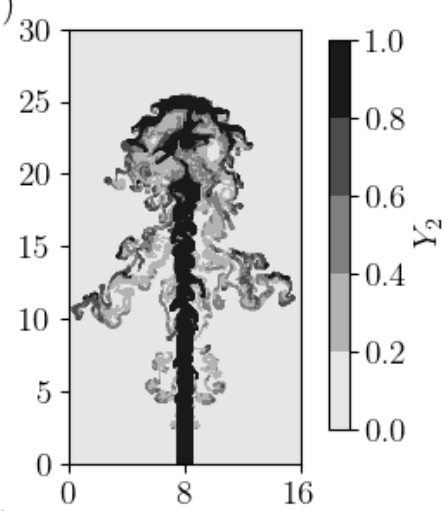

(i)

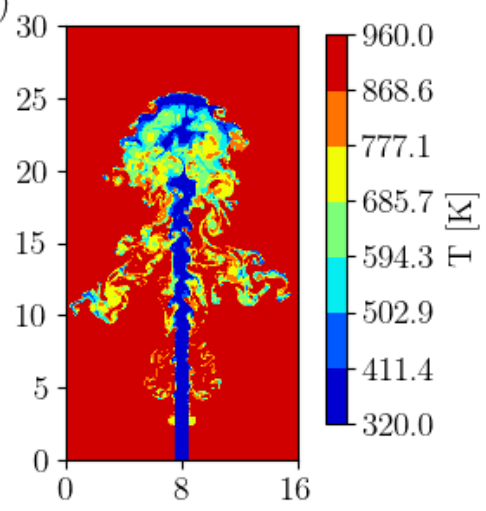

Figure 2. Comparison of the $n$-dodecane jet injection using the three numerical methods - DF $(a, d, g), H Y(b, e$, h), FC (c, f, i). (a)-(c) compares the density, (d)-(f) compares the mass fraction, and (g)-(i) compares the temperature distribution.
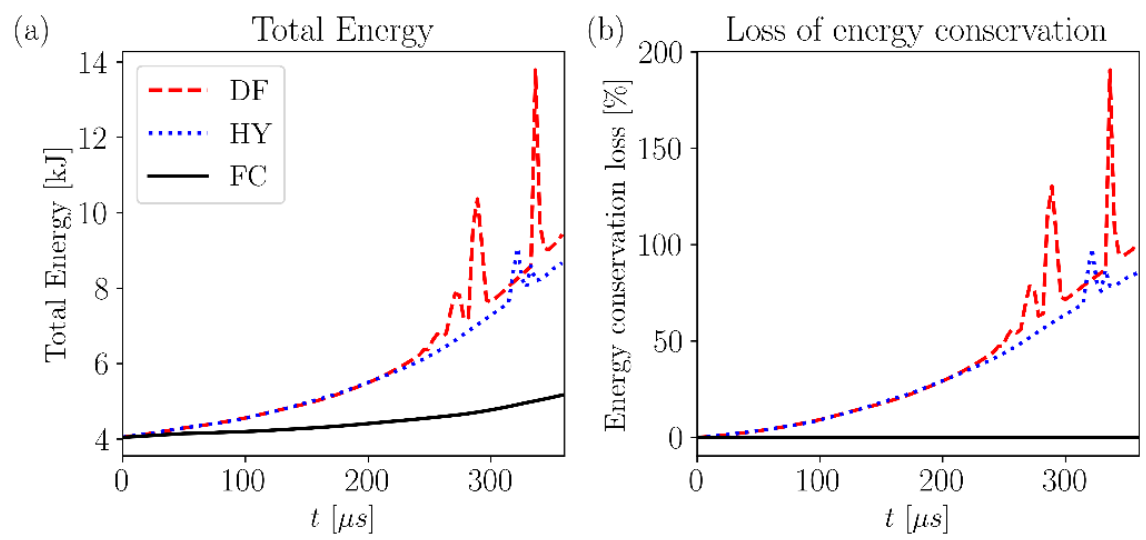

Figure 3. The temporal evolution of (a) the total energy within the domain and (b) the loss of energy conservation for the three numerical methods: DF, HY, and FC. 

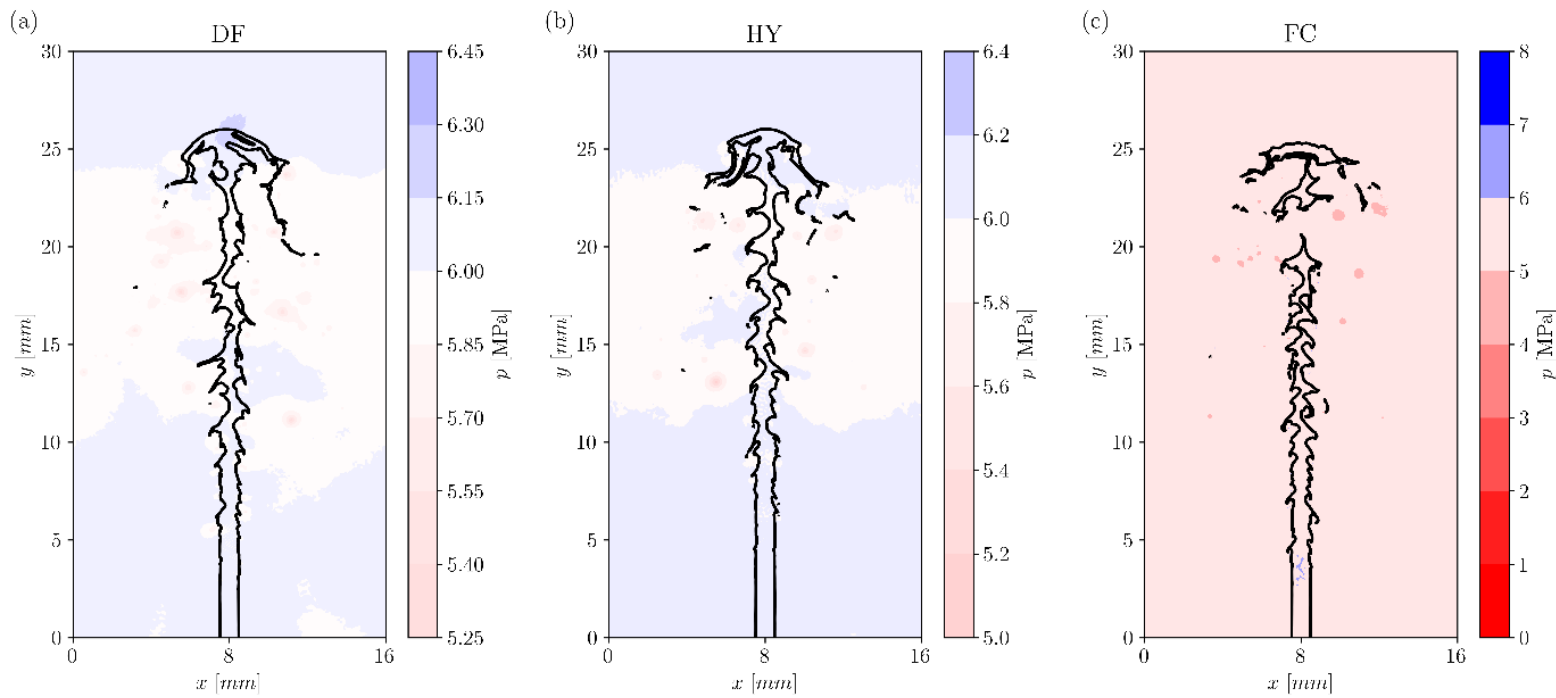

Figure 4. The pressure distribution at $360 \mu$ s for the three numerical methods: (a) DF, (b) HY, and (c) FC. The black line depicts the $Y_{2}=0.95$ contour line (species 2 is n-dodecane, i.e., $Y_{2}=1$ ).

The pressure distributions are used to show the pressure oscillations for the three numerical methods: (a) DF, (b) HY, and (c) FC. It is apparent in Figure 4 that the FC results in a larger magnitude in pressure fluctuation throughout the domain $(0-8 \mathrm{MPa})$ than the DF (5.25-6.45 $\mathrm{MPa})$ and $\mathrm{HY}$ methods (5.0-6.4 MPa). These pressure fluctuations for the FC method are slightly less than FC pressure oscillations present in Figure 7(c) of Ma et al. [21] which showed pressure fluctuations of 3.5-8.0 MPa. The DF and HY pressure fluctuations also compare well to the DF pressure oscillations present in Figure 7(d) of Ma et al. [21] which showed pressure fluctuations from about 5.0 - 6.5 MPa.

It is difficult to differentiate between the pressure fluctuations originating from the numerical instabilities and the hydrodynamically-induced pressure variations. We expect that the variations in pressure in the DF and HY method to be primarily hydrodynamically-induced as is suggested by Ma et al. [21]. The significantly larger pressure fluctuations in the FC results (Figure 4(c)) suggest that spurious pressure oscillations are present. While the similar pressure oscillations of the HY (Figure 4(b)) and DF (Figure 4(c)) suggest that the HY method is successful in mitigating the numerical pressure oscillations and yield acceptable predictions of the thermodynamic and flow parameters.

\section{Conclusions}

A finite-volume and diffuse-interface numerical method to simulate transcritical flow including single- and multi-species problems is presented. The HY method developed here combines the classic FC method with the DF method. The HY method is found to successfully mitigate spurious pressure oscillations where the FC fails. The HY method also results in less loss in energy conservation. Thus, the HY combines the benefits of the DF method (mitigating spurious pressure oscillations) and the FC method (conserving energy), making it a superior method for a larger range of problems.

\section{Acknowledgments}

Portions of this research were conducted with the advanced computing resources provided by Texas A\&M High-Performance Research Computing. 


\section{References}

1. Ma, P.C., Y. Lv, and M. Ihme, An entropy-stable hybrid scheme for simulations of transcritical real-fluid flows. Journal of Computational Physics, 2017. 340: p. 330-357.

2. Ma, P.C., et al., Large-eddy simulations of transcritical injection and auto-ignition using diffuse-interface method and finite-rate chemistry. Proceedings of the Combustion Institute, 2019. 37(3): p. 3303-3310.

3. Matheis, J. and S. Hickel, Multi-component vapor-liquid equilibrium model for LES of high-pressure fuel injection and application to ECN Spray A. International Journal of Multiphase Flow, 2018. 99: p. 294-311.

4. Ruiz, A.M., et al., Numerical Benchmark for High-Reynolds-Number Supercritical Flows with Large Density Gradients. AIAA Journal, 2015. 54(5): p. 1445-1460.

5. Schmitt, T., et al., Large-Eddy Simulation of Supercritical-Pressure Round Jets. AIAA Journal, 2010. 48(9): p. 2133-2144.

6. $\quad$ Matheis, J., et al., Large-eddy simulation of cryogenic jet injection at supercritical pressures. 2016.

7. Müller, H., et al., Large-eddy simulation of nitrogen injection at trans-and supercritical conditions. Physics of Fluids, 2016. 28(1): p. 015102.

8. Oefelein, J., R. Dahms, and G. Lacaze, Detailed modeling and simulation of high-pressure fuel injection processes in diesel engines. SAE International Journal of Engines, 2012. 5(3): p. 1410-1419.

9. Oefelein, J., et al., Effects of real-fluid thermodynamics on high-pressure fuel injection processes. SAE International Journal of Engines, 2014. 7(3): p. 1125-1136.

10. Wei, W., et al., Large eddy simulation and proper orthogonal decomposition analysis of fuel injection under trans/supercritical conditions. Computers \& Fluids, 2019. 179: p. 150-162.

11. Banuti, D.T., P.C. Ma, and M. Inme. Phase separation analysis in supercritical injection using large-eddysimulation and vapor-liquid-equilibrium. in 53rd AIAA/SAE/ASEE Joint Propulsion Conference. 2017.

12. Johnsen, E. and T. Colonius, Implementation of WENO schemes in compressible multicomponent flow problems. Journal of Computational Physics, 2006. 219(2): p. 715-732.

13. Boyd, B. and S. Becker, Numerical modelling of an acoustically-driven bubble collapse near a solid boundary. Fluid Dynamics Research, 2018. 50(6): p. 065506.

14. Boyd, B. and S. Becker, Numerical modeling of the acoustically driven growth and collapse of a cavitation bubble near a wall. Physics of Fluids, 2019. 31(3): p. 032102.

15. Zhang, X. and C.-W. Shu, Maximum-principle-satisfying and positivity-preserving high-order schemes for conservation laws: survey and new developments. Proceedings of the Royal Society A: Mathematical, Physical and Engineering Sciences, 2011. 467(2134): p. 2752-2776.

16. Qiu, J. and C.-W. Shu, On the Construction, Comparison, and Local Characteristic Decomposition for High-Order Central WENO Schemes. Journal of Computational Physics, 2002. 183(1): p. 187-209.

17. Chi-Shu, W., Essentially Non-Oscillatory and Weighted Essentially Non-Oscillatory Schemes for Hyperbolic Conservation Laws. 1997, Institute for Computer Applications in Science and Engineering (ICASE).

18. Coralic, V. and T. Colonius, Finite-volume WENO scheme for viscous compressible multicomponent flows. JOURNAL OF COMPUTATIONAL PHYSICS, 2014. 274: p. 95-121.

19. Boyd, B., Numerical modelling of an acoustically-driven bubble: the growth and collapse near a wall. 2018.

20. Knudsen, E., et al., Compressible Eulerian needle-to-target large eddy simulations of a diesel fuel injector. Proceedings of the Combustion Institute, 2017. 36(2): p. 2459-2466.

21. Ma, P.C., et al., On the numerical behavior of diffuse-interface methods for transcritical real-fluids simulations. International Journal of Multiphase Flow, 2019. 113: p. 231-249.

22. Boyd, B. and D. Jarrahbashi, A diffuse-interface method for reducing spurious pressure oscillations in multicomponent transcritical flow simulations. Computers \& Fluids, 2021: p. 104924.

23. Abgrall, R. and S. Karni, Computations of Compressible Multifluids. Journal of Computational Physics, 2001. 169(2): p. 594-623.

24. Tudisco, P. and S. Menon, Numerical Investigations of Phase-Separation During Multi-Component Mixing at Super-Critical Conditions. Flow, Turbulence and Combustion, 2020. 104(2): p. 693-724.

25. Ma, P.C., et al. Numerical analysis on mixing processes for transcritical real-fluid simulations. in 2018 AIAA Aerospace Sciences Meeting. 2018. 